\title{
COMPLETING THE BRAUER TREES FOR THE SPORADIC SIMPLE LYONS GROUP
}

\author{
JÜRGEN MÜLLER, MAX NEUNHÖFFER, FRANK RÖHR AND ROBERT WILSON
}

\begin{abstract}
In this paper, the Brauer trees are completed for the sporadic simple Lyons group Ly in characteristics 37 and 67. The results are obtained using tools from computational representation theory-in particular, a new condensation technique - and with the assistance of the computer algebra systems MeatAxe and GAP.
\end{abstract}

\section{Introduction}

\subsection{Background}

In this paper we complete the Brauer trees for the sporadic simple Lyons group Ly in characteristics 37 and 67. The results are stated in Section 2; they will also be made accessible in the character table library of the computer algebra system GAP, and are available electronically from [2]. While the shape of the Brauer trees, as well as the labelling of nodes up to algebraic conjugacy of irreducible ordinary characters, has already been described in [8, Section 6.19.], here we complete the trees by determining the labelling of the nodes on their real stems and their planar embedding; proofs are given in Section 4. Together with the results in [8, Section 6.19.] for the other primes dividing the group order, this completes all the Brauer trees for the group Ly.

Our main computational workhorse is fixed-point condensation, which was originally invented for permutation modules and described in [19], but has also been applied to other types of modules as well. To our knowledge, the permutation module that we have condensed is the largest one for which this has been accomplished so far. The theoretical background to the idea of condensation is described in Section 3. Details of the particular condensation technique used, and of some other computational tricks, are given in Section 5. For the heavy part of our computations, we have built upon and modified the implementation described in [12]. In all areas of our work, we have made substantial use of the computer algebra systems MeatAxe [17] and GAP [6]. Due to our standard setting (see Section 1.2), if we wish to write down the set of Brauer characters in the case $p=67$, we have to know the 67-modular reduction of $\sqrt{37} \in \mathbb{R}^{+}$. The latter depends on the Conway polynomial $C_{67,18}$, which was computed at our request, and is obtainable from [11]. As some tricks and heavy computation are again involved in finding $C_{67,18}$, we indicate in Section 2.2 what would be known without having $C_{67,18}$ to hand.

We note that a method similar to the one described here has also been used to solve similar, although admittedly much smaller-sized, problems for the sporadic simple Thompson group Th [4], for the sporadic simple Rudvalis group Ru and its double cover 2.Ru [18], as well as for the sporadic simple O'Nan group ON and its triple cover 3.ON [15]. The latter results 
Table 1: The Brauer tree for $p=37$.

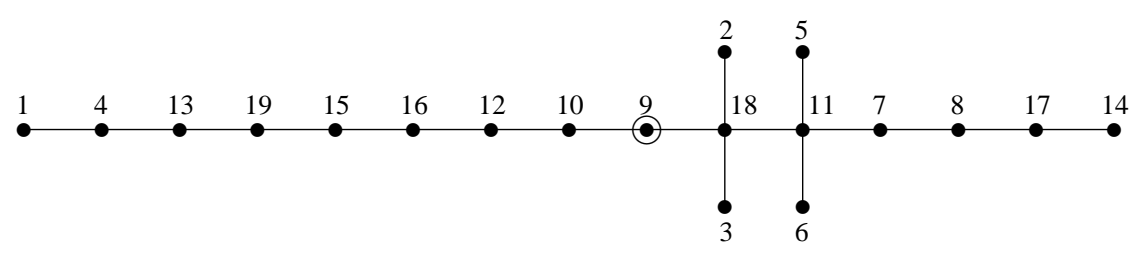

completed all the Brauer character tables for 3.ON and 2.Ru, in all the characteristics dividing each group order respectively.

\subsection{The standard setting}

We assume that the reader is familiar with block theory and the decomposition theory of finite groups, as well as with the Brauer-Dade theory of blocks of cyclic defect; see, for example, [5, Sections IV and VII].

Throughout, we use the standard choice of $p$-modular systems $(L, R, F)$ as described in [10, Introduction]. Here, $L$ is an algebraic number field, $R$ is a discrete valuation ring in $L$, with maximal ideal $\mathfrak{m} \triangleleft R$ and residue class field $F:=R / \mathfrak{m}$ of characteristic $p$. Let

$: R \rightarrow F$ denote the natural epimorphism. Assume that $\zeta_{p, n}:=\exp \left(2 \pi i /\left(p^{n}-1\right)\right) \in$ $R \subset L \subset \mathbb{C}$ for some $n \in \mathbb{N}$. Then the minimum polynomial of $\overline{\zeta_{p, n}} \in F$ over $\mathbb{F}_{p}$ is the $n$th Conway polynomial $C_{p, n} \in \mathbb{F}_{p}[X]$, where we again assume that the reader is familiar with the compatibility properties of $C_{p, n}$, when $n$ varies.

\section{Results}

In this section, we state the Brauer trees for Ly for the cases $p=37$ and $p=67$. In both cases, the principal $p$-block has defect 1 , and is the only block of positive defect. The nodes of the Brauer tree are labelled by $1,2, \ldots$, where for each case we indicate the corresponding irreducible ordinary characters of Ly according to the numbering given in [3, p. 174]. The labelling of the nodes of the Brauer trees and their planar embedding depend on the definition of the conjugacy classes of Ly in terms of the chosen pair of standard generators for Ly; see Section 4.3. For the planar embedding, we use the convention given in [8, Section 1.2.].

\subsection{The case $p=37$}

\begin{tabular}{|c|c|c|c|c|c|c|c|c|c|}
\hline Table 1 & 1 & 3 & 4 & 5 & 7 & 8 & & 9 & 10 \\
\hline [3] & 1 & 3 & 4 & 7 & 11. & 12 & 24 & 25 & 33 \\
\hline Table 1 & 11 & 12 & 13 & 14 & 15 & 16 & 17 & 18 & 19 \\
\hline & 39 & 40 & 41 & 42 & 43 & 47 & 48 & 49 & 52 \\
\hline
\end{tabular}

The exceptional node 9 has multiplicity 2 . The sets of algebraically conjugate ordinary characters are: $\{2,3\}$ and $\{5,6\}$, consisting of pairs of complex conjugate characters, and $\{11, \ldots, 15\}$ and $\{16,17\}$, consisting of real characters. The Brauer tree is given in Table 1. 
Table 2: The Brauer tree for $p=67$; for $y$ see Section 2.2.

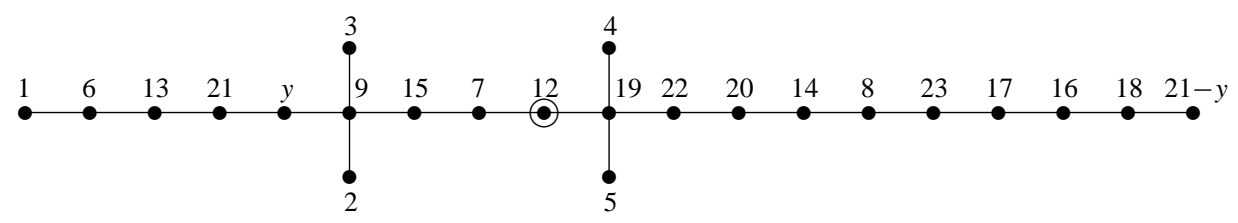

2.2. The case $p=67$

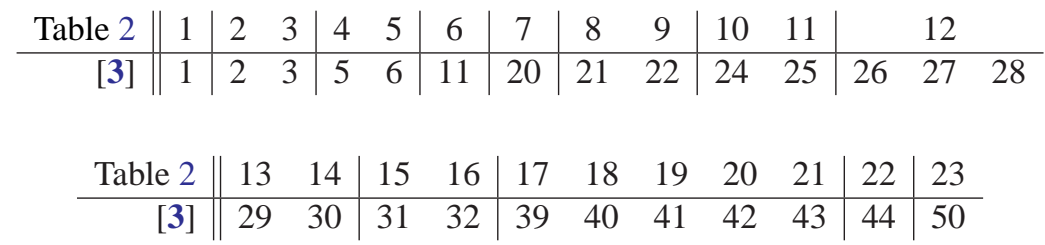

The exceptional node 12 has multiplicity 3 . The sets of algebraically conjugate ordinary characters are: $\{2,3\}$ and $\{4,5\}$, consisting of pairs of complex conjugate characters, and $\{8,9\},\{10,11\},\{13,14\},\{15,16\}$ and $\{17, \ldots, 21\}$, consisting of real characters. We remark that there is a mistake in $[8$, p. 271] concerning the relative position of the characters $\{8,9\}$ and $\{13,14\}$ on the Brauer tree. The Brauer tree is given in Table 2, where only the value of $y \in\{10,11\}$ depends on the Conway polynomial $C_{67,18}$; see Section 1 . As

$$
X^{2}-37=(X-38)(X-29) \in \mathbb{F}_{67}[X],
$$

we have $\overline{\sqrt{37}}=38 \in \mathbb{F}_{67}$ or $\overline{\sqrt{37}}=29 \in \mathbb{F}_{67}$ anyway. The former case leads to $y=10$, while the latter leads to $y=11$. Using the Conway polynomial

$$
\begin{aligned}
C_{67,18}= & 2+13 X+59 X^{2}+6 X^{3}+51 X^{4}+29 X^{5}+28 X^{6} \\
& +55 X^{7}+33 X^{8}+18 X^{9}+52 X^{10}+63 X^{11}+X^{12}+X^{18}
\end{aligned}
$$

computed in [11], we find that $\overline{\sqrt{37}}=38 \in \mathbb{F}_{67}$; hence $y=10$, for our standard choice of 67-modular system.

\section{Condensation}

\subsection{The condensation functor}

Let $\theta$ be a principal ideal domain or a field, and let $A$ be a $\theta$-algebra, which is a finitely generated $\theta$-free $\theta$-module. Let $\bmod _{\theta}-A$ be the category of finitely generated and $\theta$-free right $A$-modules, where for $V, W \in \bmod _{\theta}-A$ the homomorphism set is the set of all $\alpha \in \operatorname{Hom}_{A}(V, W)$ such that $\operatorname{im}(\alpha) \leqslant W$ is a $\theta$-pure submodule.

Let $e \in A$ be an idempotent; that is, $0 \neq e=e^{2}$. Then the additive exact functor $? \otimes_{A} A e \cong \operatorname{Hom}_{A}(e A, ?)$ from $\bmod _{\theta}-A$ to $\bmod _{\theta}-e A e$ is called the condensation functor with respect to $e$; see [7, Section 6.2]. The image $V e \in \bmod _{\theta}-e A e$ of some $V \in \bmod _{\theta}$ - $A$ under this functor is called the condensed module of $V$. Note that under this functor an $A$-homomorphism defined on $V$ is simply mapped to its restriction to $V e$. 


\subsection{Simple modules}

Let $\theta$ be a field, and let $S \in \bmod _{\theta}$-A be simple; then either $S e=\{0\}$ or $S e \in \bmod _{\theta}-e A e$ is also simple, since for $0 \neq v, v^{\prime} \in S e$ we have $v \cdot e a e=v^{\prime}$ for some $a \in A$. The following lemma shows that we can distinguish non-isomorphic simple modules in $\bmod _{\theta}-A$ by their condensed modules, if the latter are different from $\{0\}$. Note that we do not assume that $S e \neq\{0\}$ holds for all $S \in \bmod _{\theta}-A$.

Lemma. Let $\theta$ be a field, and let $S, S^{\prime} \in \bmod _{\theta}$-A be simple, such that $S e \neq\{0\} \neq S^{\prime} e$. Then $S \cong S^{\prime}$ in $\bmod _{\theta}-A$ if and only if $S e \cong S^{\prime} e$ in $\bmod _{\theta}-e$ Ae.

Proof. We need only to show the 'if' part. Assume that $S e \cong S^{\prime} e$ in $\bmod _{\theta}-e A e$, and choose a decomposition of $e$ as a sum of pairwise orthogonal primitive idempotents in $A$. As $\operatorname{Hom}_{A}(e A, S) \cong S e \neq\{0\}$, there is a summand, $e_{S}$ say, such that $e_{S} A$ is a projective indecomposable module with $e_{S} A / \operatorname{rad}\left(e_{S} A\right) \cong S$. Applying the condensation functor with respect to the idempotent $e_{S} \in e A e$, we obtain $S e_{S} \cong S^{\prime} e_{S}$ in $\bmod _{\theta}-e_{S} A e_{S}$. Hence we have $\{0\} \neq S^{\prime} e_{S} \cong \operatorname{Hom}_{A}\left(e_{S} A, S^{\prime}\right)$, and thus $S^{\prime} \cong S$ in $\bmod _{\theta}-A$.

\subsection{Reduction modulo $p$}

Let $\theta:=R$, where $R$ is as in Section 1.2. Let $A_{L}:=A \otimes_{R} L$ and $A_{F}:=A \otimes_{R} F$. If $\hat{e} \in A \subset A_{L}$ is an idempotent, then $e:=\overline{\hat{e}} \in A_{F}$ is too. The exact additive functors $\operatorname{Hom}_{A}(\hat{e} A, ?) \otimes_{R} L$ and $\operatorname{Hom}_{A_{L}}\left(\hat{e} A_{L}, ? \otimes_{R} L\right)$ from $\bmod _{R}-A$ to $\bmod _{L}-\hat{e} A_{L} \hat{e}$ are equivalent, as well as the exact additive functors $\operatorname{Hom}_{A}(\hat{e} A, ?) \otimes_{R} F$ and $\operatorname{Hom}_{A_{F}}\left(e A_{F}, ? \otimes_{R} F\right)$ from $\bmod _{R}-A$ to $\bmod _{F}-e A_{F} e$.

This means that, given $\hat{V} \in \bmod _{L}-A_{L}$ with $R$-form $V \in \bmod _{R}-A$, the $p$-modular reduction $\overline{V \hat{e}}$ of the condensed module of $V$ can be identified with the condensed module $\bar{V} e$ of the $p$-modular reduction of $V$. In this sense we speak of the condensed module in $\bmod _{F}-e A_{F}$ e of a representation of $A_{L}$.

\subsection{Fixed-point condensation}

We are going to apply a particular condensation functor, the so-called fixed-point condensation. Keeping the notation from Section 3.3, let $A=R[G]$ be the group algebra of a finite group $G$, and let $e=e_{K}:=\overline{\hat{e}} \in F[G]$, where $\hat{e}=\hat{e}_{K}:=|K|^{-1} \sum_{k \in K} k \in R[K] \subseteq R[G]$, where in turn $K$ is a subgroup of $G$ whose order $|K|$ is not divisible by the characteristic $p$ of $F$.

Let $\Omega$ be a finite set acted on by $G$, and let $F[\Omega]$ be the corresponding $F[G]$-permutation module. Then the condensed module $F[\Omega]$ e can be described as follows. Let $\left\{\Omega_{i} ; 1 \leqslant i \leqslant r\right\}$ be the set of $K$-orbits on $\Omega$, and let $\Omega_{i}^{+}:=\sum_{\omega \in \Omega_{i}} \omega \in F[\Omega]$ be the orbit sums. Then $\left\{\Omega_{i}^{+} ; 1 \leqslant i \leqslant r\right\}$ is an $F$-basis of $F[\Omega] e$, and for $g \in G$ the action of ege $\in e F[G] e$ on $F[\Omega] e$ is given as

$$
\Omega_{i}^{+} \cdot \text { ege }=\sum_{1 \leqslant j \leqslant r} a_{i j}(g) \cdot\left|\Omega_{j}\right|^{-1} \cdot \Omega_{j}^{+}, \quad \text { where } a_{i j}(g)=\left|\left\{\omega \in \Omega_{i} ; \omega g \in \Omega_{j}\right\}\right|,
$$

and where we consider $a_{i j}(g)$ and $\left|\Omega_{j}\right|$ as elements of $F$. Hence, to find the action of ege, we have to find the $K$-orbits $\left\{\Omega_{i}\right\}$, their lengths $\left|\Omega_{i}\right|$, and the $a_{i j}(g) \in \mathbb{Z}$. Note that this does not depend on the particular choice of $F$, and that an analogous description holds for $F$ replaced by $R$ or $L$, and for $e$ replaced by $\hat{e}$. 
We shall apply a new variant of fixed-point condensation of permutation modules, the socalled direct condensation technique, for which the basic idea was conceived by R. Parker and R. Wilson. The technique was subsequently modified, extending its range of applicability, in [12]. Here, we have built upon and modified the latter implementation; see Sections 4.4 and 5.2.

\subsection{The dimension formula}

Let $V \in \bmod _{F}-F[G]$, and let $\varphi_{V}$ be its Brauer character, viewed as an $L$-valued class function on the $p^{\prime}$-classes of $G$. Then $\varphi_{V}$ can be extended to a class function $\tilde{\varphi}_{V}$ on the whole of $G$ as follows. For $g \in G$, let $g_{p}, g_{p^{\prime}} \in G$ denote its $p$-part and its $p^{\prime}$-part, respectively, and let $\tilde{\varphi}_{V}(g)=\varphi_{V}\left(g_{p^{\prime}}\right)$. As $F[K]$ is a semisimple algebra, we have $\operatorname{dim}_{F}(V e)=\left\langle\left(\varphi_{V}\right)_{K}, 1_{K}\right\rangle_{K}=\left\langle\tilde{\varphi}_{V}, 1_{K}^{G}\right\rangle_{G}$, where $\langle\cdot, \cdot\rangle_{G}$ denotes the usual scalar product for class functions on $G$.

Let $W \in \bmod _{L}-L[G]$, and let $\chi_{W}$ be its ordinary character. In view of the remarks in Section 3.3, we have $\operatorname{dim}_{F}(\bar{W} e)=\operatorname{dim}_{L}(W \tilde{e})=\left\langle\left(\chi_{W}\right)_{K}, 1_{K}\right\rangle_{K}=\left\langle\chi_{W}, 1_{K}^{G}\right\rangle_{G}$. Writing $\varphi_{V}$ as a $\mathbb{Z}$-linear combination of ordinary characters restricted to the $p^{\prime}$-classes of $G$, the dimension $\operatorname{dim}_{F}(V e)$ can be computed in terms of ordinary characters. As the blocks under consideration here are described by Brauer trees, these linear combinations can be read off directly from the trees.

\subsection{The trace formula}

To solve the algebraic conjugacy problems, we apply the following idea. Let $V \in$ $\bmod _{F}-F[G]$, and let $\varphi_{V}$ be its Brauer character. If $t_{V}$ and $t_{V e}$ are the usual $F$-valued trace functions, we have

$$
t_{V e}(e g e)=|K|^{-1} \cdot \sum_{k \in K} t_{V}(g k)=|K|^{-1} \cdot \sum_{k \in K} \overline{\tilde{\varphi}_{V}(g k)} .
$$

If $V e$ is explicitly given, the trace $t_{V e}(e g e)$ can be evaluated explicitly as well. On the other hand, $t_{V e}(e g e)$ can be computed if $\varphi_{V}$ is known, and if it is known to which conjugacy classes of $G$ the elements of the coset $g K$ belong.

\section{Proofs}

\subsection{A permutation representation}

Let $G=$ Ly for short. We are looking for a permutation representation of $G$, such that its permutation character has sufficiently many constituents belonging to the principal $p$-blocks, for both the cases $p=37$ and $p=67$. Let $5^{3}: L_{3}(5) \cong H<G$ be a maximal subgroup of index 1113229656 (see [3, p. 174]), let $\Omega$ be the set of right cosets of $H$ in $G$, and let $\chi_{\Omega}$ be the character of $\mathbb{C}[\Omega]$. Note that neither $p=37$ nor $p=67$ divides the group order $|H|$, and hence $\mathbb{F}_{37}[\Omega]$ and $\mathbb{F}_{67}[\Omega]$ are projective $\mathbb{F}_{37}[G]$ - and $\mathbb{F}_{67}[G]$-modules, respectively.

Let $V$ be the absolutely irreducible 111 -dimensional representation of $G$ over $\mathbb{F}_{5}$. This was constructed in [14], and is accessible electronically from [21], as representing matrices for a pair $g=\left\{g_{1}, g_{2}\right\}$ of standard generators, in the sense of [20]. In [21] we also find words in $g$ yielding a subgroup conjugate to $H$. These were obtained by a random search among the subgroups generated by an involution and an element of class $3 A$; see [3, p. 174]. We find the submodule structure of the restriction $V_{H}$ of $V$ to $H$, using the algorithms in [13], 
Table 3: Characters, multiplicities and condensed dimensions.

\begin{tabular}{r|rr}
$\chi$ & $m$ & $d$ \\
\hline 1 & 1 & 1 \\
2 & $\cdot$ & $\cdot$ \\
3 &. & $\cdot$ \\
4 & 1 & 4 \\
5 &. & 1 \\
6 &. & 1 \\
7 &. &. \\
8 &. & $\cdot$ \\
9 & 1 & 1 \\
10 &. & 5 \\
11 & 2 & 14 \\
12 & 1 & 20 \\
13 &. & 9 \\
14 & 2 & 18
\end{tabular}

\begin{tabular}{c|rr}
$\chi$ & $m$ & $d$ \\
\hline 15 & $\cdot$ & 24 \\
16 & $\cdot$ & 16 \\
17 & $\cdot$ & 25 \\
18 & 1 & 32 \\
19 & $\cdot$ & 48 \\
20 & 1 & 47 \\
21 & 1 & 57 \\
22 & 1 & 57 \\
23 & 1 & 52 \\
24 & 1 & 56 \\
25 & 1 & 56 \\
26 & 1 & 56 \\
27 & 1 & 56
\end{tabular}

\begin{tabular}{r|rr}
$\chi$ & $m$ & $d$ \\
\hline 28 & 1 & 56 \\
29 & 2 & 88 \\
30 & 2 & 88 \\
31 & 1 & 82 \\
32 & 1 & 82 \\
33 & 2 & 81 \\
34 & 1 & 87 \\
35 & $\cdot$ & 78 \\
36 & 2 & 109 \\
37 & $\cdot$ & 102 \\
38 & 1 & 98 \\
39 & 1 & 120 \\
40 & 1 & 120
\end{tabular}

\begin{tabular}{c|rr}
$\chi$ & $m$ & $d$ \\
\hline 41 & 1 & 120 \\
42 & 1 & 120 \\
43 & 1 & 120 \\
44 & $\cdot$ & 116 \\
45 & $\cdot$ & 110 \\
46 & 1 & 126 \\
47 & 1 & 133 \\
48 & 1 & 133 \\
49 & $\cdot$ & 140 \\
50 & $\cdot$ & 148 \\
51 & 1 & 151 \\
52 & 1 & 172 \\
53 & 1 & 196
\end{tabular}

implemented in the MeatAxe. In particular, it turns out that $V_{H}$ has a simple socle $S$ of dimension 10. As $H<G$ is a maximal subgroup and $G$ acts irreducibly on $V$, we conclude that $H$ is the stabilizer in $G$ of $S$. Thus the action of $G$ on the set $S^{G}$ of subspaces of $V$ is equivalent to its action on $\Omega$, and we may identify $\Omega$ with $S^{G}$.

\subsection{A condensation subgroup}

As we are going to condense the permutation module $F[\Omega]$ of dimension $\sim 10^{9}$, we need a condensation subgroup of order at least $\sim 10^{5}$ to obtain a condensed module of a dimension small enough that its structure can be analysed using the MeatAxe. Here, a subgroup 2. $A_{9} \cong K<G$, having order 362880 , springs to mind. In particular, $K$ is contained in a maximal subgoup $2 . A_{11} \cong L<G$, for which we also find a generating set as words in $g$ in [21]. Such words are found, for example, by using the method described in [1] for finding involution centralizers. We then find standard generators for $L$, which are preimages of standard generators of $A_{11}$. The latter are $A_{11}$-conjugate to the pair $\{(1,2,3),(3,4,5,6,7,8,9,10,11)\}$ in the natural permutation representation of $A_{11}$. From that, generators $\left\{k_{1}, k_{2}\right\}$ for $K$ are found as words in $g$.

Using the ordinary character tables of $H, K$ and $G$, accessible in GAP, and its library functions dealing with conjugacy class fusions and scalar products between characters, the fusions of the conjugacy classes of $H$ and $K$ into those of $G$ are determined, as well as the multiplicities $m$ of the irreducible ordinary characters $\chi$ of $G$ in $\chi_{\Omega}$. Taking into account the remarks in Section 3.5, the dimensions $d$ of the condensed modules of the irreducible ordinary representations of $G$, with respect to the condensation subgroup $K$, can also be computed as scalar products. The results are given in Table 3. In particular, the condensed module $F[\Omega] e$, where $e=e_{K}$, has dimension 3207, independent of the particular choice of $F$. 


\section{Brauer trees for Ly}

Table 4: Definition of the conjugacy classes of Ly.

\begin{tabular}{|c|c|c|c|c|c|c|}
\hline & $\tilde{\varphi}_{V}$ & $\tilde{\varphi}_{W}$ & $\tilde{\varphi}_{X}$ & $V$, over $\mathbb{F}_{5}$ & $W$, over $\mathbb{F}_{3}$ & $X$, over $\mathbb{F}_{5}$ \\
\hline $1 A$ & 111 & 651 & 2480 & & & \\
\hline $2 A$ & -1 & 11 & -16 & & & \\
\hline $3 A$ & -24 & 651 & 104 & $t(g)=1$ & & \\
\hline $3 B$ & 3 & 651 & -4 & $t(g)=3$ & & \\
\hline $4 A$ & 3 & -5 & 0 & & & \\
\hline $5 A$ & 111 & 26 & -20 & $t(g)=1$ & $t(g)=2$ & \\
\hline $5 B$ & 111 & 1 & 5 & $t(g)=1$ & $t(g)=1$ & \\
\hline $6 A$ & 8 & 11 & 8 & $t(g)=3$ & $r\left((g-1)^{2}\right)=426$ & \\
\hline $6 B$ & -1 & 11 & -4 & $t(g)=4$ & $r\left((g-1)^{2}\right)=430$ & \\
\hline $6 C$ & -1 & 11 & 2 & $t(g)=4$ & $r\left((g-1)^{2}\right)=429$ & \\
\hline $7 A$ & -1 & 0 & 2 & & & \\
\hline $8 A$ & -3 & -1 & 0 & $t(g)=2$ & & \\
\hline $8 B$ & 1 & -1 & 0 & $t(g)=1$ & & \\
\hline $9 A$ & 0 & 651 & -1 & & & \\
\hline $10 A$ & -1 & 6 & 4 & $t(g)=4$ & $t(g)=0$ & \\
\hline $10 B$ & -1 & 1 & -1 & $t(g)=4$ & $t(g)=1$ & \\
\hline $11 A$ & 1 & 2 & $b_{11}$ & & & $t(g)=1$ \\
\hline $11 B$ & 1 & 2 & ** & & & $t(g)=3$ \\
\hline $12 A$ & 0 & -5 & 0 & $t(g)=0$ & & \\
\hline $12 B$ & -3 & -5 & 0 & $t(g)=2$ & & \\
\hline $14 A$ & -1 & 4 & -2 & & & \\
\hline $15 A$ & -24 & 26 & 4 & $t(g)=1$ & $t(g)=2$ & \\
\hline $15 B$ & 3 & 26 & 1 & $t(g)=3$ & $t(g)=2$ & \\
\hline $15 C$ & -24 & 1 & -1 & $t(g)=1$ & $t(g)=1$ & \\
\hline $18 A$ & 2 & 11 & -1 & & & \\
\hline $20 A$ & 3 & 0 & 0 & & & \\
\hline $21 A$ & $*$ & 0 & -1 & $t(g)=1$ & & \\
\hline $21 B$ & $-b_{21}$ & 0 & -1 & $t(g)=0$ & & \\
\hline $22 A$ & -1 & 0 & $-b_{11}$ & & & $t(g)=4$ \\
\hline $22 B$ & -1 & 0 & $* *$ & & & $t(g)=2$ \\
\hline $24 A$ & 0 & -1 & 0 & $t(g)=0, t\left(g^{2}\right)=0$ & & \\
\hline $24 B$ & $1+r_{6}$ & -1 & 0 & $t(g)=0, t\left(g^{2}\right)=2$ & & \\
\hline $24 C$ & * & -1 & 0 & $t(g)=2, t\left(g^{2}\right)=2$ & & \\
\hline $25 A$ & 111 & 1 & 0 & & & \\
\hline $28 A$ & 3 & 2 & 0 & & & \\
\hline $30 A$ & 8 & 6 & -2 & $t(g)=3$ & & \\
\hline $30 B$ & -1 & 6 & 1 & $t(g)=4$ & & \\
\hline $31 A$ & $e_{31} * 4 \& 8 \& 16$ & 0 & 0 & $t(g)=1, t\left(g^{2}\right)=4$ & & \\
\hline $31 B$ & $* 2$ & 0 & 0 & $t(g)=4, t\left(g^{2}\right)=3$ & & \\
\hline $31 C$ & $* 4$ & 0 & 0 & $t(g)=3, t\left(g^{2}\right)=2$ & & \\
\hline $31 D$ & $* 8$ & 0 & 0 & $t(g)=2, t\left(g^{2}\right)=2$ & & \\
\hline $31 E$ & $* 16$ & 0 & 0 & $t(g)=2, t\left(g^{2}\right)=1$ & & \\
\hline $33 A$ & -2 & 2 & $b_{11}$ & & & $t(g)=1$ \\
\hline $33 B$ & -2 & 2 & ** & & & $t(g)=3$ \\
\hline $37 A$ & 0 & $4+b_{37}$ & 1 & $t(g)=0$ & $t(g)=1$ & \\
\hline $37 B$ & 0 & $*$ & 1 & $t(g)=0$ & $t(g)=0$ & \\
\hline $40 A$ & -3 & $4+r_{10}$ & 0 & $t(g)=2$ & $t(g)=2$ & \\
\hline $40 B$ & -3 & $*$ & 0 & $t(g)=2$ & $t(g)=0$ & \\
\hline $42 A$ & $-3-b_{21}$ & 4 & 1 & $t(g)=2$ & & \\
\hline $42 B$ & $*$ & 4 & 1 & $t(g)=3$ & & \\
\hline $67 A$ & $-1-c_{67}$ & $3-c_{67}$ & 1 & $t(g)=1$ & & \\
\hline $67 B$ & $* 2$ & $* 2$ & 1 & $t(g)=3$ & & \\
\hline $67 C$ & $* 4$ & $* 4$ & 1 & $t(g)=4$ & & \\
\hline
\end{tabular}


Table 5: The class distribution of $g_{3} K$

\begin{tabular}{|c|c|c|c|c|c|c|c|}
\hline class & \# & class & \# & class & \# & class & \# \\
\hline $1 A$ & 0 & $10 A$ & 95 & $21 B$ & 8663 & $31 D$ & 11721 \\
\hline $2 A$ & 0 & $10 B$ & 7092 & $22 A$ & 16387 & $31 E$ & 11659 \\
\hline $3 A$ & 0 & $11 A$ & 5406 & $22 B$ & 16657 & $33 A$ & 10935 \\
\hline $3 B$ & 0 & $11 B$ & 5496 & $24 A$ & 15173 & $33 B$ & 11115 \\
\hline $4 A$ & 16 & $12 A$ & 1205 & $24 B$ & 15054 & $37 A$ & 9927 \\
\hline $5 A$ & 0 & $12 B$ & 5067 & $24 C$ & 15194 & $37 B$ & 9775 \\
\hline $5 B$ & 89 & $14 A$ & 2106 & $25 A$ & 14522 & $40 A$ & 9183 \\
\hline $6 A$ & 4 & $15 A$ & 172 & $28 A$ & 13023 & $40 B$ & 9008 \\
\hline $6 B$ & 172 & $15 B$ & 3976 & $30 A$ & 3975 & $42 A$ & 8707 \\
\hline $6 C$ & 1105 & $15 C$ & 4891 & $30 B$ & 4088 & $42 B$ & 8589 \\
\hline $7 A$ & 2172 & $18 A$ & 20394 & $31 \mathrm{~A}$ & 11561 & $67 A$ & 5413 \\
\hline $8 A$ & 765 & $20 A$ & 9035 & $31 B$ & 11660 & $67 B$ & 5269 \\
\hline $8 B$ & 3776 & $21 A$ & 8767 & $31 C$ & 11731 & $67 C$ & 5429 \\
\hline $9 A$ & 6661 & & & & & & \\
\hline
\end{tabular}

\subsection{A class distribution}

Later on, we shall apply the formula given in Section 3.6 to the element $g_{3}:=$ $\left(g_{1} g_{2}\right)^{3} g_{2} \in G$, which has order 67 . We have to find the distribution of the elements in the coset $g_{3} \mathrm{~K}$ into the conjugacy classes of $G$.

The conjugacy classes of cyclic subgroups of $G$ are defined by group-theoretic datathat is, by element orders, centralizer orders, and normalizer orders, as can be checked in [3, p. 174]. Words in $g$ giving representatives for the conjugacy classes of cyclic subgroups are accessible from [21]. For our purposes, we have to distinguish algebraically conjugate classes. To do this, we use traces and ranks of certain elements of $\mathbb{Z}[G]$ on $V$, as well as traces and ranks on the absolutely irreducible 651-dimensional representation $W$ over $\mathbb{F}_{3}$, which was constructed in [9] and is accessible from [21], and on one of the algebraically conjugate 2480-dimensional absolutely irreducible representations $X, \tilde{X}$ over $\mathbb{F}_{5}$, which are 5 -modular reductions of ordinary representations. The latter have been constructed for the purposes of this paper, using the MeatAxe, as constituents of the exterior square of $V$; they are also accessible from [21]. To distinguish $X$ and $\tilde{X}$, we define the conjugacy class $22 A$ of $G$ to be the class containing the particular element of order 22 given in [21] as a word in $g$. Using this, $X$ is the 5-modular reduction of the representation affording the ordinary character $\chi_{2}$.

The details are given in Table 4, where $t$ and $r$ denote the trace and the rank, respectively, and where we also give the values of the corresponding extended Brauer characters (see Section 3.5) using the notation of [3]. We find the numbers of elements belonging to the different conjugacy classes as shown in Table 5; for details of the computations, see Section 5.1.

\subsection{Applying condensation}

We condense the elements $g_{1}, g_{2}$ and $g_{3}$, where $g=\left\{g_{1}, g_{2}\right\}$ is as in Section 4.1, and where $g_{3}=\left(g_{1} g_{2}\right)^{3} g_{2} \in G$ is as in Section 4.3; for details of the computations, see 
Table 6: Partial information for $p=37$; see Section 4.5 .

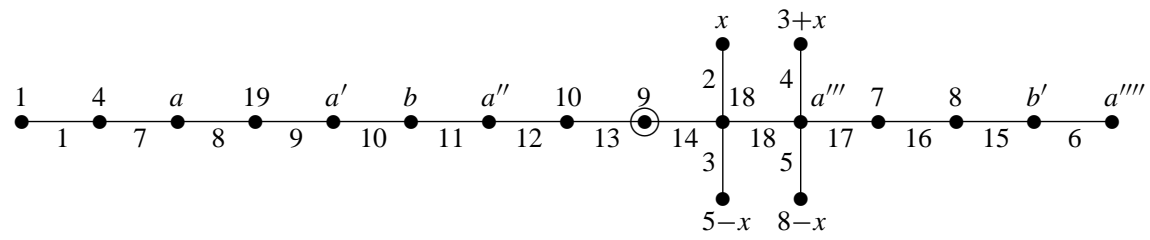

Table 7: Brauer characters and condensed dimensions for $p=37$.

\begin{tabular}{r|rr}
$\varphi$ & $m$ & $d$ \\
\hline 1 & 2 & 1 \\
2 & $\cdot$ & $\cdot$ \\
3 & $\cdot$ & $\cdot$ \\
4 & 1 & $\cdot$ \\
5 & 1 & $\cdot$ \\
6 & 2 & 120
\end{tabular}

\begin{tabular}{r|rr}
$\varphi$ & $m$ & $d$ \\
\hline 7 & 2 & 3 \\
8 & 2 & 117 \\
9 & 2 & 55 \\
10 & 2 & 65 \\
11 & 2 & 68 \\
12 & 3 & 52
\end{tabular}

\begin{tabular}{r|rr}
$\varphi$ & $m$ & $d$ \\
\hline 13 & 4 & 29 \\
14 & 2 & 27 \\
15 & 2 & 13 \\
16 & 3 & 7 \\
17 & 3 & 7 \\
18 & 1 & 113
\end{tabular}

Section 5.2. Note that by Section 3.4 this essentially amounts to finding the $a_{i j}(g) \in \mathbb{Z}$; hence we do not have to specify the field $F$ beforehand.

\subsection{The case $p=37$}

The partial information on the Brauer tree known from [8, p. 268] is shown in Table 6, where $\left\{x, x^{\prime}\right\}=\{2,3\}$, and $\left\{a, a^{\prime}, a^{\prime \prime}, a^{\prime \prime \prime}, a^{\prime \prime \prime \prime}\right\}=\{11, \ldots, 15\}$, and $\left\{b, b^{\prime}\right\}=\{16,17\}$. Hence there are 480 possible cases left. We also give labels to the edges of the Brauer tree, for future reference. The dimensions $d$ of the condensed modules of the irreducible modular representations of $G$ can be computed from this information; for those in the principal block they are given in Table 7, where the numbering is as given in the Brauer tree in Table 6. We also give the multiplicities $m$ of the corresponding Brauer characters in the principal block component of the permutation character $\chi_{\Omega}$. The dimensions of the condensed modules of the representations not in the principal block, and the multiplicities of the corresponding ordinary irreducible characters, have already been given in Table 3.

We now specify $F:=\mathbb{F}_{37}$. Using the MeatAxe, the condensed module $F[\Omega] e$, acted on by the $F$-algebra $F[\mathcal{E}]$ generated by $\mathcal{E}:=\left\{e g_{1} e, e g_{2} e, e g_{3} e\right\}$, turns out to have the following constituents, where we denote the constituents by their dimension and a trailing letter, and their multiplicities by exponents:

$$
\begin{gathered}
1 a^{2}, 1 b^{1}, 3 a^{2}, 7 a^{3}, 7 b^{3}, 13 a^{2}, 18 a^{2}, 27 a^{2}, 29 a^{4}, 32 a^{1}, 47 a^{1}, \\
52 a^{3}, 52 b^{1}, 55 a^{2}, 57 a^{1}, 57 b^{1}, 65 a^{2}, 68 a^{2}, 87 a^{1}, 88 a^{2}, 88 b^{2}, 98 a^{1}, \\
109 a^{2}, 113 a^{1}, 117 a^{2}, 120 a^{2}, 126 a^{1}, 151 a^{1}, 164 a^{1}, 168 a^{1}, 196 a^{1} .
\end{gathered}
$$


Here the constituents $164 a$ and $168 a$ split over $\mathbb{F}_{37^{2}}$ and $\mathbb{F}_{37^{3}}$, respectively, while all the others are absolutely irreducible.

If $\varphi$ is an irreducible Brauer character occurring in $\chi_{\Omega}$, which is afforded by the simple $F[G]$-module $S_{\varphi}$, then either $S_{\varphi} e$ is equal to $\{0\}$, or it is a simple $e F[G] e$-module; see Section 3.1. But as we have $F[\Omega]$ e given as an $F[\mathcal{E}]$-module only, where $F[\mathcal{E}]$ might be strictly smaller than $e F[G] e$, we can only try to determine the constituents of $\left.\left(S_{\varphi} e\right)\right|_{F[\mathcal{E}]}$ and their multiplicities. This is done by comparing the dimensions and multiplicities of the constituents of $F[\Omega] e$ found by the MeatAxe, using the data given in Tables 7 and 3. These considerations show that for all $S_{\varphi} e \neq\{0\}$, the restriction $\left.\left(S_{\varphi} e\right)\right|_{F[\mathcal{E}]}$ is simple as well, and that this indeed gives a bijection from the set of irreducible Brauer characters $\varphi$ occurring in $\chi_{\Omega}$, such that $S_{\varphi} e \neq\{0\}$, and the constituents found by the MeatAxe. Furthermore, this bijection is uniquely determined, up to the fact that it maps $\left\{\varphi_{16}, \varphi_{17}\right\}$ to $\{7 a, 7 b\}$.

Recall that $F[\Omega]$ is a projective $F[G]$-module. By decomposing $\chi_{\Omega}$ into projective indecomposable characters and using the multiplicities given in Table 7 , we conclude that in a given decomposition of $F[\Omega]$ into projective indecomposable summands, both the projective covers $P_{16}$ of $\varphi_{16}$ and $P_{17}$ of $\varphi_{17}$ occur with multiplicity 1 . Furthermore, none of the other projective indecomposable summands has $\varphi_{16}$ or $\varphi_{17}$ as a constituent. The MeatAxe, together with the peakword technique described in [13], shows that there is an $F[\mathcal{E}]$-submodule $U_{1} \leqslant\left(P_{16} \oplus P_{17}\right) e$ of dimension 34 , having a simple head and a simple socle isomorphic to $7 a$, and containing the constituent $7 b$ with multiplicity 1 , and an $F[\mathcal{E}]$-submodule $U_{2} \leqslant\left(P_{16} \oplus P_{17}\right) e$ of dimension 134 having a simple head and a simple socle isomorphic to $7 b$ and containing the constituent $7 a$ with multiplicity 1 . As $P_{16} e$ and $P_{17} e$ have dimensions 34 and 134, respectively, we have $U_{1} \oplus U_{2}=\left(P_{16} \oplus P_{17}\right) e$. By the Krull-Schmidt theorem, we conclude that $\left.U_{1} \cong\left(P_{16} e\right)\right|_{F[\mathcal{E}]}$ and $\left.U_{2} \cong\left(P_{17} e\right)\right|_{F[\mathscr{E}]}$. Thus the above bijection maps $\varphi_{16}$ to $7 a$ and $\varphi_{17}$ to $7 b$.

We find the following traces $t_{S_{\varphi}}\left(e g_{3} e\right) \in F$ of the action of $e g_{3} e$ :

\begin{tabular}{r||rrrrrrrrrrrrrrrrrr}
$\varphi$ & 1 & 2 & 3 & 4 & 5 & 6 & 7 & 8 & 9 & 10 & 11 & 12 & 13 & 14 & 15 & 16 & 17 & 18 \\
\hline$t_{S_{\varphi} e}$ & 1 &. &. &. &. & 10 & 1 & 36 & 5 & 9 & 14 & 19 & 13 & 20 & 36 & 25 & 29 & 6
\end{tabular}

This gives the left-hand side of the trace formula in Section 3.6 for the condensed modules corresponding to the $\varphi_{i}$. Using the class distribution found in Section 4.3, we compute the right-hand side of the formula for the remaining 480 cases. It turns out that there are precisely two cases that are consistent with the actual traces found by the MeatAxe; these are the Brauer tree printed in Section 2.1, for which we have $x=2$, and the tree obtained by reflecting the first case at its real stem, for which we have $x=3$. Hence it remains to determine the planar embedding of the Brauer tree.

\subsection{The planar embedding}

We still let $F:=\mathbb{F}_{37}$. As both the pairs $\varphi_{1,2}$ and $\varphi_{3,4}$ condense to the zero module, the condensation subgroup $K$ is not suitable for us to use to determine the planar embedding of the Brauer tree. Instead, we use another condensation subgroup $\tilde{K} \cong 2 .\left(A_{6} \times A_{5}\right) .2$ of order 86400, which is also contained in the maximal subgroup $2 . A_{11} \cong L<G$, and we repeat the steps described in Sections 4.2, 4.3 and 4.4. The condensed module $F[\Omega] \tilde{e}$, where $\tilde{e}=e_{\tilde{K}}$, has dimension 13257 . Note that (due to this large dimension) it would not have been feasible to analyse $F[\Omega] \tilde{e}$, instead of $F[\Omega] e$, as completely as was necessary to find the labelling of the nodes of the Brauer tree in Section 4.5.

In particular, we find that $S_{\varphi_{16}} \tilde{e}, S_{\varphi_{17}} \tilde{e}$ and $S_{\varphi_{18}} \tilde{e}$ have dimensions 16, 20 and 477, 
Table 8: Partial information for $p=67$, see Section 4.7.

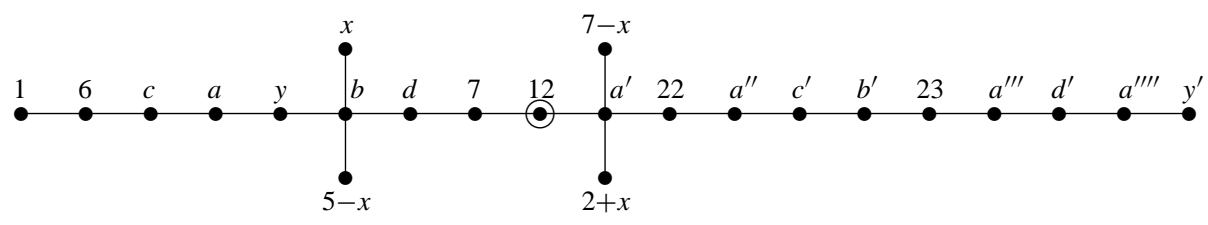

respectively, and that $S_{\overline{\chi_{7}}} \tilde{e}$ and $S_{\overline{\chi_{8}}} \tilde{e}$ both have dimension 1 , where $\overline{\chi_{7}}$ and $\overline{\chi_{8}}$ denote the 37-modular reductions of the ordinary characters $\chi_{7}$ and $\chi_{8}$; see Section 3.3. Furthermore, $\tilde{e} g_{3} \tilde{e}$ acts by multiplication with $21 \in F$ and $26 \in F$ on $S_{\overline{\chi_{7}}} \tilde{e}$ and $S_{\overline{\chi_{8}}} \tilde{e}$, respectively. In a given decomposition of $F[\Omega]$ into projective indecomposable summands, $P_{17}$ occurs with multiplicity 1 , and none of the other projective indecomposable summands has $\overline{\chi_{7}}$ or $\overline{\chi_{8}}$ as a constituent. Hence the constituents $S_{\overline{\chi_{7}}} \tilde{e}$ and $S_{\overline{\chi_{8}}} \tilde{e}$ of $F[\Omega] \tilde{e}$ occur with multiplicity 1 , and are constituents of the $\tilde{e} F[G] \tilde{e}$-submodule $P_{17} \tilde{e} \leqslant F[\Omega] \tilde{e}$. By the submodule structure of projective indecomposable modules in blocks of cyclic defect, there is a unique minimal submodule $U \leqslant P_{17} \tilde{e}$ having both $S_{\overline{\chi_{7}}} \tilde{e}$ and $S_{\overline{\chi_{8}}} \tilde{e}$ as constituents. Furthermore, $U$ has dimension 499 , and is uniserial with the ascending composition series $S_{\varphi_{17}} \tilde{e}, S_{\varphi_{5}} \tilde{e}, S_{\varphi_{18}} \tilde{e}, S_{\varphi_{4}} \tilde{e}$.

We consider $F[\Omega] \tilde{e}$ as a module for the $F$-algebra $F[\tilde{\mathscr{E}}]$ being generated by $\tilde{\mathcal{E}}:=$ $\left\{\tilde{e} g_{1} \tilde{e}, \tilde{e} g_{3} \tilde{e}\right\}$, and we let $N:=\operatorname{ker}_{F[\Omega] \tilde{e}}\left(\tilde{e} g_{3} \tilde{e}-21 \tilde{e}\right)$. The MeatAxe finds that $N$ has dimension 1 , that the $F[\tilde{\varepsilon}]$-module $\tilde{N}:=N \cdot F[\tilde{\mathcal{E}}] \leqslant F[\Omega] \tilde{e}$ has dimension 499, and that it is uniserial with the ascending composition series $20 a, 1 a, 477 a, 1 b$, where $\tilde{e} g_{3} \tilde{e}$ acts by multiplication with $26 \in F$ and $21 \in F$ on $1 a$ and $1 b$, respectively. Furthermore, the MeatAxe finds that $\operatorname{ker}_{F[\Omega] \tilde{e} / \tilde{N}}\left(\tilde{e} g_{3} \tilde{e}-21 \tilde{e}\right)=\{0\}$, and hence $\tilde{e} g_{3} \tilde{e}-21 \tilde{e} \in F[\tilde{\mathcal{E}}]$ is a peakword on $F[\Omega] \tilde{e}$ for the constituent $1 b$; see [13]. From that we conclude that $\tilde{N}$ is contained in $U$, and hence $\tilde{N}=U$ is an $\tilde{e} F[G] \tilde{e}$-submodule of $P_{17} \tilde{e}$. Thus we have $\left.\left.\left(S_{\overline{\chi_{7}}} \tilde{e}\right)\right|_{F[\tilde{\mathcal{E}}]} \cong 1 b \cong\left(S_{\varphi_{4}} \tilde{e}\right)\right|_{F[\tilde{\mathcal{E}}]}$, and hence $x=2$.

\subsection{The case $p=67$}

The partial information on the Brauer tree known from [8, p. 271] is shown in Table 8, where

$$
\begin{aligned}
\left\{x, x^{\prime}\right\} & =\{2,3\} ; \\
\left\{b, b^{\prime}\right\} & =\{8,9\} ; \\
\left\{y, y^{\prime}\right\} & =\{10,11\} ; \\
\left\{c, c^{\prime}\right\} & =\{13,14\} ; \\
\left\{d, d^{\prime}\right\} & =\{15,16\} ; \\
\left\{a, a^{\prime}, a^{\prime \prime}, a^{\prime \prime \prime}, a^{\prime \prime \prime \prime}\right\} & =\{17, \ldots, 21\} .
\end{aligned}
$$

Hence there are 3840 possible cases left. We find the Brauer tree by analysing $F[\Omega] e$, where we now specify that $F=\mathbb{F}_{67}$, using similar techniques to those described in Sections 4.5 and 4.6; again, we omit the details here. 
Table 9: Modified orbit algorithm.

$$
\begin{aligned}
& T:=[1] ; \\
& \text { for } g \text { in } T \text { do } \\
& \text { for } k \text { in } \mathcal{K} \text { do } \\
& h:=g \cdot k ; \\
& \text { if } h \text { not in } T \text { then } \\
& \text { Append } h \text { to } T ; \\
& i:=1 ; \\
& \text { while } i \leqslant t \text { do } \\
& h^{\prime}:=h \cdot k_{i} ; \\
& \text { if } h^{\prime} \text { not in } T \text { then } \\
& \text { Append } h^{\prime} \text { to } T ; \\
& h:=h^{\prime} ; \\
& i:=1 ; \\
& \text { else } \\
& i:=i+1 ;
\end{aligned}
$$

\section{Comments on the computations}

In this section we give some details of how the computations were done. We concentrate on the calculations for the condensation subgroup $K$; see Sections 4.3 and 4.4. The condensation subgroup $\tilde{K}$ (see Section 4.6) was treated similarly.

\subsection{Finding the class distribution}

As described in Section 4.3, we have to determine how the elements of the coset $g_{3} K$ distribute into the conjugacy classes of $G$. To do this, we have to compute representing matrices for the $|K|=362880$ elements in the coset $g_{3} K$ on the modules $V, W$ and $X$, and then to compute some traces or ranks. However, to keep $|K|$ many $(2480 \times 2480)$-matrices over $\mathbb{F}_{5}$ in memory would require $\sim 7.5 \times 10^{11}$ bytes; hence we want to obtain a reasonable number of matrices, which have to be stored simultaneously.

Let $\mathcal{K}=\left\{k_{1}, \ldots, k_{t}\right\}$ be a fixed generating set for $K$. In a precomputation, we use an orbit algorithm to enumerate the elements of $K$, starting with $1 \in K$. This yields a Schreier tree for $K$ with respect to $\mathcal{K}$, whose shape depends on the particular strategy employed in the orbit algorithm. Given a Schreier tree, we define a valuation $v$ on its vertices as follows. If the vertex $g$ is a leaf, we let $v(g)=1$. Otherwise, if $g_{1}, \ldots, g_{s}$ are the immediate successors of $g$ in the Schreier tree, we let $v(g)=\max \left\{v\left(g_{i}\right) ; 1 \leqslant i \leqslant s\right\}$ if this maximum is assumed exactly once, and $v(g)=1+\max \left\{v\left(g_{i}\right) ; 1 \leqslant i \leqslant s\right\}$ if it is assumed more than once. In the recursive run through the Schreier tree, used to find the class distribution, we are now able at each vertex to work through the most expensive subtree last. Hence, for each matrix representation being considered, we have to store no more than $v(1)$ matrices at the same time, in addition to the representing matrices for $\mathcal{K}$. Our aim is thus to find a Schreier tree such that $v(1)$ is reasonably small.

To find a suitable Schreier tree, we enumerate a regular $K$-orbit in $V$, as its elements are in bijection with the elements of $K$. The algorithm that we use is a modification of the PubCrawl algorithm presented in [16, Section 5]; see Table 9. Note that $T$ is a list that collects the orbit during the algorithm. New vectors are appended to $T$ within the loop that 
runs through $T$. Of course, the loop must also run through these new vectors. The idea is to use a standard breadth-first orbit algorithm as an outer loop to run through the whole orbit, but each time we find a new element of the orbit (that is, a new vertex of the Schreier tree), before going on with the standard algorithm we try to attach a 'thread' (that is, a lengthy path without branching points) to the corresponding vertex in the Schreier tree.

We choose the generating set $\mathcal{K}$ as follows. We fix a 2-Sylow subgroup $\tilde{U}$ of $K$ as a helper subgroup (hence $|\tilde{U}|=2^{7}$ ), and we let $\mathcal{K}$ be the union of the generating set $\left\{k_{1}, k_{2}\right\}$ of $K$ (see Section 4.2) and a set of generators of $\tilde{U}$. Thus we end up with a Schreier tree such that $v(1)=3$.

As $|\tilde{U}|$ is coprime to the characteristics of $V, W$ and $X$, we choose bases exhibiting the semisimplicity of $\left.V\right|_{\tilde{U}},\left.W\right|_{\tilde{U}}$ and $\left.X\right|_{\tilde{U}}$. As the constituents of these modules have dimension at most 8 , this considerably reduces the amount of time needed for a matrix multiplication with one of the generators of $\tilde{U}$. Furthermore, using the MeatAxe and the algorithms in [13], $\left.X\right|_{K}$ turns out to be a direct sum of 14 indecomposable modules, the largest of which has dimension 560. We therefore adapt the basis of $X$, such that it also exhibits a direct sum decomposition of $\left.X\right|_{K}$.

Building the Schreier tree, running through the elements of $g_{3} K$, and computing the representing matrices, traces and ranks is easily done using the new fast finite field arithmetic of GAP, which is based on the ideas of the arithmetic of the MeatAxe. Using a Pentium III 800 processor, this needed $\sim 60$ hours of CPU time.

\subsection{Applying condensation}

As described in Section 4.4, we want to compute the action of the elements $\left\{e g_{1} e, e g_{2} e\right.$, $e g_{3} e$ \} on the condensed module $F[\Omega] e$, where the permutation module $F[\Omega]$ is given by the action of $\left\{g_{1}, g_{2}\right\}$ on the set $\Omega=S^{G}$ of subspaces of $V$ of dimension 10; see Section 4.1. As $\Omega$ is not yet known, it has to be enumerated first, and we subsequently have to compute the integers $a_{i j}(g)$ - see 3.4-for all $\omega \in \Omega$ and $g \in\left\{g_{1}, g_{2}, g_{3}\right\}$. But to store a subspace of $V$ of dimension 10 we need 370 bytes, and thus to store the whole orbit $\Omega$ of length $[G: H]$ we would need $\sim 4 \times 10^{11}$ bytes. Hence we can only afford to store $\sim 1 / 400$, say, of the elements of $\Omega$, which would fit into $\sim 10^{9}$ bytes. We use a modification of the ideas expounded in [12].

We choose a helper subgroup $U \leqslant K \leqslant G$, such that its elements can be enumerated, and objects representing their action on $\Omega$ can be kept in memory. The basic idea is now to modify the standard breadth-first orbit algorithm for $G$, such that $\Omega$ is enumerated piecewise, namely $K$-orbit by $K$-orbit, where these are in turn enumerated $U$-orbit by $U$-orbit, keeping track of how the $U$-orbits fall into $K$-orbits. Hence, for $\omega \in \Omega$, we are reduced to finding out whether we have already encountered its orbit $\omega^{U}$ and, if this is the case, finding which it is of the orbits that have already been encountered.

To do this, we choose a helper $U$-set $\Theta$, such that there is a homomorphism $q:\left.\Omega\right|_{U} \rightarrow \Theta$ of $U$-sets. Furthermore, we assume that the elements of $\Theta$ can be enumerated completely. Let $\left\{\Theta_{i} ; 1 \leqslant i \leqslant s\right\}$ be the $U$-orbits on $\Theta$. For each $1 \leqslant i \leqslant s$, we choose a minimal element $\vartheta_{i} \in \Theta_{i}$ (for example, by using an injective function on $\Theta$ into a totally ordered set, which is quickly evaluated on the elements of $\Theta$ ). An element $\omega \in \Omega$ is called $q$-minimal if $q(\omega)$ is minimal. It is exactly the $q$-minimal elements that are stored in a table during the enumeration of the whole of $\Omega$; to recover elements quickly, we of course use a hashing technique. If we are able to find a homomorphism $q$ such that most of the $\left\{\Theta_{i}\right\}$ as above are regular $U$-orbits, then $\sim 1 /|U|$ of the elements of $\Omega$ will be $q$-minimal. We shall thus 
be content in this case with a subgroup $U$ of order $|U| \sim 400$.

Furthermore, let $\kappa: \Theta \rightarrow \mathcal{P}(U)$, where $\mathcal{P}(U)$ denotes the power set of $U$, be defined as follows. For $\vartheta \in \Theta_{i}$, we let $\kappa(\vartheta)=\left\{u \in U ; \vartheta \cdot u=\vartheta_{i}\right\}$, which is therefore a left coset of $\operatorname{Stab}_{U}\left(\vartheta_{i}\right)$ in $U$. Since we assume that the elements of $U$ and those of $\Theta$ can be enumerated, we are able to compute all the sets $\kappa(\vartheta)$ explicitly. If we want to check, for some $\omega \in \Omega$, whether the orbit $\omega^{U}$ has been encountered before, we try to look up $\omega \cdot u$, for an arbitrary $u \in \kappa(q(\omega))$, in the table. If it is there, we have encountered $\omega^{U}$ before; if it is not, we store all of the elements $\{\omega \cdot u ; u \in \kappa(q(\omega))\}$ in the table.

Having fixed the subgroup $U$, we try to find a suitable $U$-set $\Theta$. We look for $U$ modules $X_{1}$ and $X_{2}$, each of dimension 11, such that there are epimorphisms of $U$-modules $q_{i}: V_{U} \rightarrow X_{i}$, and $\operatorname{ker}\left(q_{1}\right) \cap \operatorname{ker}\left(q_{2}\right)<V$ is of codimension 22. Let $\Theta_{i}$ be the set of all subspaces of $X_{i}$ of codimension 1 , and let $\Theta_{2}^{\prime}$ be the set of all proper subspaces of $X_{2}$ of codimension at least 2. As $\Omega$ consists of subspaces of $V$ of dimension 10 , there is a homomorphism of $U$-sets $q: \Omega \rightarrow \Theta:=\Theta_{1} \cup \Theta_{2} \cup \Theta_{2}^{\prime}$, defined by $q(\omega)=q_{1}(\omega)$ if $q_{1}(\omega) \in \Theta_{1}$, and $q(\omega)=q_{2}(\omega)$ otherwise.

A simple calculation shows the reason for this choice. As $X_{i}$ has dimension 11 over $\mathbb{F}_{5}$, there are $\left(5^{11}-1\right) /(5-1) \sim 1.2 \times 10^{7}$ and $\left(5^{11}-1\right)\left(5^{11}-5\right) /\left(\left(5^{2}-1\right)\left(5^{2}-5\right)\right) \sim 5 \times 10^{12}$ subspaces of $X_{i}$ of codimension 1 and 2 , respectively, and even more of higher codimension. Thus it is possible to enumerate only the subspaces of $X_{i}$ of codimension 1 , and hence the above-mentioned preprocessing is done only for $\Theta_{1} \cup \Theta_{2}$. By going over to the dual space $X_{i}^{*}=\operatorname{Hom}_{\mathbb{F}_{5}}\left(X_{i}, \mathbb{F}_{5}\right)$, we need 4 bytes to store one of these subspaces of $X_{i}$, which still requires $\sim 9.8 \times 10^{7}$ bytes for the whole of $\Theta_{1} \cup \Theta_{2}$. This means that all the subspaces of $V$ mapped by $q$ into $\Theta_{1} \cup \Theta_{2}$ are dealt with as explained above, but those mapped into $\Theta_{2}^{\prime}$ simply have to be stored, and so for these we save no memory at all. The memory requirements are estimated as follows.

There are $\prod_{i=0}^{9}\left(5^{111}-5^{i}\right) /\left(5^{10}-5^{i}\right)$ subspaces of $V$ of dimension 10 , but of these, only $\prod_{i=0}^{9}\left(5^{111}-5^{100+i}\right) /\left(5^{10}-5^{i}\right)$ intersect trivially with $\operatorname{ker}\left(q_{1}\right)$. Hence $\sim 1 / 20$ of these subspaces are not mapped into $\Theta_{1}$ by $q_{1}$. If we assume $\Omega$ to consist of a uniformly distributed random sample of subspaces of $V$ of dimension 10, this amounts to $\sim 1 / 20 \cdot[G: H] \sim 5.5 \times 10^{7}$ subspaces. To store this many subspaces, at the cost of 370 bytes each, we would need $\sim 2 \times 10^{10}$ bytes. This shows the need for a second map $q_{2}$. Then only $\sim 1 / 400$ of the elements of $\Omega$ are expected to be mapped by $q$ into $\Theta_{2}^{\prime}$, which requires $\sim 2.7 \times 10^{6}$ elements or $\sim 10^{9}$ bytes, which still means that we need as much memory for these elements of $\Omega$ as for its $q$-minimal elements. We remark that the memory requirements in the actual computations did indeed fit well into this picture.

Despite these serious constraints, we were lucky to find a suitable maximal subgroup $3^{2}: 2 A_{4} \cong \bar{U}<A_{9} \cong K / Z(K)$ of order 216 . It is uniquely defined up to conjugacy in $A_{9}$; see [3, p. 37]. We let $Z(K) \times \bar{U} \cong U<K$ be the preimage of $\bar{U}$ with respect to the natural epimorphism $K \rightarrow A_{9}$; hence $|U|=432$. Again, we chose a basis for $V$ exhibiting the semisimplicity of $\left.V\right|_{U}$. As the constituents of $\left.V\right|_{U}$ have dimension at most 16 , this considerably reduces the amount of time needed for a matrix multiplication with one of the elements of $U$. Using the MeatAxe, together with the algorithms in [13], we found epimorphic images $X_{1}$ and $X_{2}$ of $V_{U}$, where $X_{1} \cong 1^{-} \oplus 2 \oplus 8$ and $X_{2} \cong 3 \oplus 8^{-}$. Here $Z(K)$ acts non-trivially on the constituents $1^{-}$and $8^{-}$, and trivially on the others. Hence $U$ acts faithfully on both $X_{1}$ and $X_{2}$, and $Z(K)$ acts non-trivially on subspaces of $X_{1}$ and $X_{2}$ of codimension 1 . The average orbit length of $U$ on subspaces of codimension 1 turned out to be $\sim 405$ for $X_{1}$, and $\sim 415$ for $X_{2}$. 
With these preparations, we accordingly adjusted the implementation described in [12], which allows for massive parallelization. We are grateful to the University of St. Andrews for allowing us to use their PC cluster to run these computations. Using 50 Pentium II 450 processors, the computations needed $\sim 13$ hours of elapsed time, and hence $\sim 650$ hours of CPU time.

Acknowledgements. We thank the University of St. Andrews for allowing us to use their PC cluster, under EPSRC grant GR/M 32351. The first three authors thank the fourth author and the University of Birmingham for their hospitality, and the DAAD for financial support. The fourth author thanks the first three authors and the RWTH Aachen for their hospitality, and the British Council for financial support.

\section{References}

1. J. BraY, 'An improved method for generating the centraliser of an involution', Arch. Math. 74 (2000) 241-245. 23

2. T. Breuer et al., 'The modular atlas', http://www. math.rwth-aachen.de/ moc. 18

3. J. Conway, R. Curtis, S. Norton, R. Parker and R. Wilson, Atlas of finite groups (Clarendon Press, 1985). 19, 19, 19, 20, 20, 22, 22, 25, 25, 31

4. G. Cooperman, G. Hiss, K. Lux and J. Müller, 'The Brauer tree of the principal 19-block of the sporadic simple Thompson group', Experiment. Math. 6 (1997) 293-300. 18

5. W. FEIT, The representation theory of finite groups (North-Holland, 1982). 19

6. The GAP Group, GAP - Groups, Algorithms, and Programming, Version 4.2 (Aachen/St. Andrews, 2000); http: / /www.gap-system.org/. 18

7. J. Green, Polynomial representations of $G L_{n}$, Lecture Notes in Math. 830 (Springer, 1980). 20

8. G. Hiss and K. Lux, Brauer trees of sporadic groups (Clarendon Press, 1989). 18 , $18,19,20,26,28$

9. C. JANSEN and R. WiLSON, 'The minimal faithful 3-modular representation of the Lyons group', Comm. Algebra 24 (1996) 873-879. 25

10. C. JANSEN, K. LuX, R. PARKER and R. WiLson, An atlas of Brauer characters (Clarendon Press, 1995). 19

11. F. LÜBECK, 'Conway ploynomials of finite fields';

http: / / www . math. rwth-aachen. de/ Frank. Luebeck/ConwayPol/ 18,20

12. F. LÜBECK and M. NEUNHÖFFER, 'Enumerating large orbits and direct condensation', Experiment. Math. 10 (2001) 197-205. 18, 22, 30, 32

13. K. Lux, J. Müller and M. Ringe, 'Peakword condensation and submodule lattices: an application of the MeatAxe', J. Symb. Comp. 17 (1994) 529-544. 22, 27, 28, 30, 31

14. W. Meyer, W. Neutsch and R. PArker, 'The minimal 5-representation of Lyons' sporadic group', Math. Ann. 272 (1985) 29-39. 22 


\section{Brauer trees for Ly}

15. M. OtTEnsmann, 'Vervollständigung der Brauer-Bäume von $3 . O N$ in Charakteristik 11, 19 und 31 mit Methoden der Kondensation', Diplomarbeit, Lehrstuhl D für Mathematik, RWTH Aachen, 2000. 18

16. R. PARKer and R. Wilson, 'Constructions of Fischer's Baby Monster over fields of characteristic not 2', J. Algebra 229 (2000) 109-117. 29

17. M. Ringe, The C-MeatAxe Manual, Version 2.4 (Lehrstuhl D für Mathematik, RWTH Aachen, 2000). 18

18. F. RöHr, 'Die Brauer-Charaktere der sporadischen einfachen Rudvalis-Gruppe in Charakteristik 13 und 29', Diplomarbeit, Lehrstuhl D für Mathematik, RWTH Aachen, 2000. 18

19. J. ThaCKRAY, 'Modular representations of some finite groups', PhD thesis, Cambridge University, 1981. 18

20. R. WiLson, 'Standard generators for sporadic simple groups', J. Algebra 184 (1996) 505-515. 22

21. R. Wilson et al., 'Atlas of finite group representations', http: //www.mat.bham.ac.uk/atlas/. 22, 22, 23, 25, 25, 25, 25

Jürgen Müller mueller@math.rwth-aachen.de

Max Neunhöffer max.neunhoeffer@math.rwth-aachen.de

Lehrstuhl D für Mathematik

RWTH Aachen

Templergraben 64

D-52062 Aachen

Germany

Frank Röhr roehr@modell.iig.uni-freiburg.de

Institut für Informatik und Gesellschaft

Abteilung Modellbildung und soziale Folgen

Universität Freiburg

D-79085 Freiburg i.Br.

Germany

Robert Wilson R.A.Wilsonebham.ac.uk

School of Mathematics and Statistics

The University of Birmingham

Edgbaston

Birmingham B15 2TT 